Rapport - Société canadienne d'histoire de l'Église catholique

\title{
Le Journal des Jésuites de Québec de 1632 à 1645
}

\section{Léon Pouliot}

Volume 28, 1961

URI : https://id.erudit.org/iderudit/1007377ar

DOI : https://doi.org/10.7202/1007377ar

Aller au sommaire du numéro

Éditeur(s)

La Société canadienne d'histoire de l'Église catholique

ISSN

0318-6148 (imprimé)

1927-7075 (numérique)

Découvrir la revue

Citer cet article

Pouliot, L. (1961). Le Journal des Jésuites de Québec de 1632 à 1645. Rapport -

Société canadienne d'histoire de l'Église catholique, 28, 45-52.

https://doi.org/10.7202/1007377ar

Tous droits réservés @ La Société canadienne d'histoire de l'Église catholique, 1963
Ce document est protégé par la loi sur le droit d'auteur. L'utilisation des services d’Érudit (y compris la reproduction) est assujettie à sa politique d'utilisation que vous pouvez consulter en ligne.

https://apropos.erudit.org/fr/usagers/politique-dutilisation/ 


\title{
Le Journal des Jésuites de Québec
}

\author{
de 1632 à 1645
}

Bien qu'il n'existe aucune prescription explicite sur le sujet, un usage s'est établi dans la Compagnie de Jésus, et dès les débuts, suivant lequel tout supérieur local tient un diaire ou journal: entrée au jour le jour des événements qui tranchent sur le quotidien et qui appartiennent à l'histoire intérieure ou extérieure de la maison. Cet usage découle, croyons-nous, d'un devoir imposé par saint Ignace à ses enfants, dans la huitième partie des Constitutions : devoir pour les supérieurs locaux de renseigner régulièrement le Provincial sur les hommes et les œuvres de leur maison, devoir pour le Provincial d'être en relation épistolaire constante avec Rome. Par là saint Ignace pensait moins à exercer un contrôle minutieux sur ses fils qu'à resserrer les liens de la charité entre des frères, séparés dans l'espace, mais engagés dans le même combat. Il voulait également stimuler le zèle des uns par le récit des résultats obtenus ailleurs par les autres ${ }^{1}$.

De tant de documents parvenus à Rome, il appartiendrait au Général de tirer des extraits, de les publier et de les communiquer à toute la Compagnie. A une époque où il n'existait ni presse quotidienne, ni téléphone, ni télégraphe, où les communications d'un pays à l'autre étaient lentes et difficiles, on avait recours au livre pour renseigner les Jésuites du monde entier sur les travaux de leurs frères. Cela nous a valu l'importante collection des Littera Annuce, ou Lettres annuelles, manuscrites d'abord, mais imprimées depuis la fin du XVI' siècle ${ }^{2}$.

Comme on le voit, dans la pensée de saint Ignace, la correspondance n'était pas pure et vaine formalité. Elle avait un but pratique et un but apostolique; tout en tenant le Provincial au courant, elle était collaboration, par l'exemple, à l'œuvre de toute la Compagnie. Et c'est pour une plus parfaite observation de cette règle qu'a été conçu l'aide-mémoire, qui s'appelle le Journal. Si les supérieurs des maisons d'Europe avaient cru bon, entre deux rapports au Provincial, de confier à un Journal les faits et les événements dignes de retenir l'attention, on pense bien qu'il n'en fut pas autrement en Nouvelle-France. Bien plus, indépendamment des bonnes coutumes de France, que le père Paul Le Jeune apportait avec lui en 1632, et qu'il avait à cour de maintenir, le besoin d'un Journal se faisait plus impérieusement sentir ici qu'en Europe; car la courte navigation fluviale d'alors ne permettait au Supérieur de Québec de s'entretenir qu'une fois l'année avec le Provincial de Paris. Quand, après les longs mois d'hiver, l'heure viendrait

1 Societatis Jesu Constitutiones, Rome 1949, p. 243, nn. 673-676.

2 Les Lettres annuelles n'étaient pas destinées au public. - Etude sur les Relations des Jésuites... Montréal, 1940, 4-5. 
de rédiger le rapport ou Relation, la tâche du Supérieur serait singulièrement facilitée, s'il avait à la main cet aide-mémoire qui s'appelle le Journal. Mais à Québec, ce Journal a-t-il vraiment existé ? et s'il a existé, quand a-t-il commencé ?

En 1871, les abbés Laverdière et Casgrain publiaient, d'après le manuscrit original, conservé aux archives du Séminaire de Québec, le Journal des Jésuites de Québec de 1645 à $1668^{3}$. Ils savaient que la suite du Journal avait existé jusqu'en 1755, qu'elle avait été connue

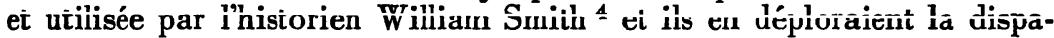
rition. Mais, en se basant sur le titre du manuscrit : Journal commencé, octobre 1645, ils insinuaient, sans pourtant l'affirmer explicitement, qu'il n'y avait rien de semblable pour les années antérieures ${ }^{5}$. L'usage constant de la Compagnie, que nous venons de rappeler, la connaissance plus approfondie que nous avons aujourd'hui du père Paul Le Jeune, premier Supérieur de Québec ${ }^{6}$, la lecture attentive des Relations que nous poursuivons depuis plus de vingt ans nous permettent d'affirmer que le Journal a commencé dès 1632 , et qu'il est digne de foi, aux mêmes titres que celui du père Lalemant. Peu importe, après cela, le sort qu'a eu, dans la suite des temps, le Journal des Jésuites de Québec de 1632 à 1645; peu importe qu'il ait été rédigé sur des feuilles volantes ou dans un cahier solide, qu'il ait été détruit par le feu ou qu'il ait été considéré par ses auteurs mêmes comme document négligeable, une fois publiée la Relation, qui en communiquait l'essen-

3 In-IV de 403 pages, Léger Brousseau, Québec. - Cette première édition fut presque tout entière consumée par le feu avant d'être mise en vente. Les rares exemplaires qui ont survécu font l'orgueil des bibliophiles. En 1892, le libraire-éditeur de Montréal, J.M. Valois, publiait une seconde édition exactement conforme à la première. - Le Journal des Jésuites n'existe plus en librairie depuis de longues années. Qui se chargera de nous en donner une troisième édition? - Dans ses Jesuit Relations and allied documents, Thwaites reproduit le Journal année par année, à partir de 1645, selon l'ordre chronologique rigoureux de la collection. Il nous paraît regrettable qu'un document d'une telle importance n'ait pas été publié d'une seule venue.

4 The History of Canada from its discovery to the peace of 1763. Québec, Neilson, 1815. Le premier volume, p. 170 , contient une référence au Journal des Jésuites, à la date du 20 décembre 1710. Dans le même volume, p. 223, une autre référence pour l'année 1752, mais sans indication plus précise. Les éditeurs du Journal ajoutent : . M. Faribault, qui paraît avoir eu communication des papiers de $M$. Smith, nous apprend que cet écrivain en a fait quelques extraits de l'année 1710 à 1755. J Journal, IX. Mais où sont et que sont devenus les papiers de William Smith?

5 . Ce précieux manuscrit paraît être le premier journal régulier qu'aient tenu les Jésuites du Canada; nous en trouvons une forte preuve dans le titre même..., Journal, VII.

6 Après trois siècles d'oubli, le père Paul Le Jeune connaît aujourd'hui une juste popularité. En novembre 1957, dans la collection Classiques canadiens, que publie Fides, paraissait un choix de textes empruntés aux Relations. Un mois plus tard, le jeune Jésuite, Gilles Chaussé, présentait à la Faculté des Lettres de l'Université de Montréal une thèse de maîtrise : Le père Paul Le Jeune, missionnaire-colonisateur. Au début de janvier de 1958, le père Jean Bouchard, s.j. soutenait devant la Faculté de Missiologie de l'Université Grégorienne une thèse de doctorat : Les méthodes missionnaires du père Paul Le Jeune. Nous avons eu l'avantage de consulter ces deux thèses et nous en remercions les auteurs. 
tiel et qui avait été sa principale raison d'être. Il a existé; et, en ce qu'il affirme, il est digne de foi. Voilà pourquoi nous ne craignons pas d'intituler cet ouvrage : Les Premières Pages du Journal des Jésuites de Québec, 1632-1645.

Il ne s'agit donc pas de la découverte sensationnelle d'un manuscrit, qui aurait échappé jusqu'ici à l'attention des chercheurs; il s'agit d'un ensemble de faits, dispersés dans les Relations, mais dont le caractère, l'exactitude et la présentation ne s'expliquent que par cet aidemémoire qui s'appelle le Journal. En d'autres termes, de même que le Journal a servi à la rédaction des Relations, de même nous nous servons aujourd'hui des Relations pour reconstituer le Journal. Entrons en discours, comme dirait le père Paul Le Jeune.

Le plus sérieux, sinon le seul argument apporté contre l'existence d'un Journal antérieur à 1645 se trouve dans le titre du manuscrit : Journal commencé, octobre 1645. L'argument nous paraîtrait plus convaincant, s'il se plaçait au milieu de l'administration du père Jérôme Lalemant; mais comme il coïncide avec l'entrée en charge du nouveau Supérieur, il peut tout aussi bien signifier qu'il s'agit d'un cahier nouveau que d'une innovation proprement dite. Nous verrons plus bas que le père Lalemant avait des raisons particulières de confier ses entrées à un cahier solide, destiné à prendre place dans la bibliothèque du Supérieur; souci que n'aurait pas eu le père Le Jeune. Mais ici, soyons prudents : il est possible et il nous paraît fort vraisemblable que le Journal du père Le Jeune ait été irrémédiablement perdu dans l'incendie du 14 juin $1640^{7}$. Pour le moment, contentons-nous de nous demander si le Journal des Jésuites de Québec a existé de 1632 à 1645.

Dans notre Etude sur les Relations des Jésuites de la Nouvelle$F_{\text {rance }}{ }^{8}$ et dans notre Paul Le Jeune ${ }^{9}$, nous avons affirmé, et personne n'y a contredit, que la Briève Relation de 1632 n'était rien autre chose que le Journal de voyage du père Le Jeune, rédigé à l'adresse du Provincial de Paris, en accomplissement de la règle.

Au printemps de 1633 , le 22 mai exactement, il apprend que la Briève Relation a connu à Paris les honneurs de la publication, et qu'elle a suscité un véritable enthousiasme. Il comprend qu'il $y$ a là une veine à exploiter pour le bien de la mission qui lui est confiée, qu'il faut donner plus d'ampleur au rapport annuel ou Relation. Mais, il n'est pas pris au dépourvu; il a sous la main une matière abondante et curieuse. Comment la présente-t-il ? a Afin d'éviter la confusion, je suivrai l'ordre du temps ${ }^{10}$. \ Après une brève digression sur la colonie française, il reprend, pour ne plus se laisser distraire, cette fois : « Mais venons au départ des vaisseaux, l'an passé, pour suivre les mois qui

7 Voir plus bas cette date, dans le Journal reconstitué.

8 Collection Studia. Etudes publiées par le scolasticat de l'Immaculée-Conception de Montréal, 1940, XII-319.

9 Classiques canadiens, Fides, Montréal, 1957, 96 pages.

10 Q. 1633, 2; Th. V, 84. 
se sont écoulés depuis ce temps-là ${ }^{11}$. $\mathrm{Et}$, du 10 octobre 1632 au 6 août 1633, les faits sont datés avec précision, mais n'ont d'autre lien entre eux que celui du temps. A qui fera-t-on croire que le père Le Jeune a daté les événements post factum et au hasard, comme pour donner plus d'autorité à son récit? Pour qui connaît sa probité, une seule conclusion s'impose : les dates indiquées dans la Relation sont exactes. Elles viennent en ligne directe du Journal.

Avec 1634, la Relation atteint sa forme définitive : a Je distinguerai la Relation de cette année par chapitre, à la fin desquels je mettrai un journal des choses qui n'ont autre liaison que ía suite des temps auxquels elles sont arrivées ${ }^{12}$. "Ainsi, dans un texte où le père Le Jeune manifeste son intention de s'adresser désormais au public, non à son seul Provincial, il affirme explicitement l'existence du Journal. Et le chapitre XIII, contenant un journal des choses qui n'ont pu être couchées sous les chapitres précédents ${ }^{13}$, a deux parties distinctes, mais qui viennent de la même source : son Journal. C'est là qu'il puise les principales dates de son hivernement avec les Sauvages, ainsi que le nombre de fois qu'ils ont cabané et décabané. Après avoir justifié cette longue narration : "Retournons à notre journal », écrit-il ${ }^{14}$. Et c'est, avec leurs dates, la suite des principaux événements survenus à Québec depuis son retour.

De 1635 à 1640 inclusivement, la Relation contient un chapitre intitulé : Ramas ou Journal de diverses choses. En le lisant, on a l'impression, voire la certitude, d'assister avec le père Le Jeune au dépouillement de son Journal. Il omet les faits qu'il a pu logiquement placer ailleurs : il retient les autres et il nous les présente dans leur ordre chronologique. Si ce Ramas ou Journal va s'amincissant d'année en année, c'est que l'accoutumance et l'habitude du milieu ont émoussé, chez le père Le Jeune, la faculté d'émotion. Il n'est plus l'étranger, qui considère comme étrange tout ce qui n'est pas conforme aux us et coutumes de l'Ancienne France; il est parfaitement assimilé, incorporé à la Nouvelle. Mais, si restreint qu'il soit, l'existance de ce Ramas et la façon dont il est présenté, attestent la continuité du Journal des Jésuites jusqu'à 1640 inclusivement.

Pour les années 1641-1645, nous n'avons pas la certitude que le Journal ait été tenu avec la même régularité. On peut être bon religieux et bon supérieur sans avoir au même degré que le père Le Jeune. le sens de l'observation et la passion de l'écriture. Et il semble que ce soit le cas du père Barthélemy Vimont. Il s'était déchargé sur son prédécesseur du soin de la Relation, et par là, pensons-nous, du Journal. Et nous savons que le père Le Jeune passe en France les hivers de $1641-164,2$ et de 1643-1644.14 b1s

1 Ibid., Th. V, 86.

12 Q. 1634, 2; Th. VI, 100.

13 Ibid., 57; Th. VII, 66.

14 Ibid., 87; Th. VII, 210.

14 bis Il ne faudrait pas conclure trop vite que le Journal du père Vimont n'a jamais existé. A défaut d'autres preuves, les chapitres VII, VIII et IX de la Relation de 1645 notent plusieurs faits, datés avec précision et qui se rapportent à la fin de 1644 ou au début de 1645 , date à laquelle le père Jérôme Lalemant n'est pas encore à Québec. 
Il n'en reste pas moins que pendant ces années 1641-1645, il s'est trouvé à Québec, aux Trois-Rivières, à Richelieu et à Montréal des Pères de la Compagnie de Jésus pour consigner par écrit, à l'intention des Supérieurs et des lecteurs des Relations, les principaux événements avec leurs dates. En insérant ces faits dans le Journal des Jésuites de Québec, nous ne faisons qu'imiter la ligne de conduite adoptée par le père Jérôme Lalemant lui-même. Le 8 janvier 1646, pour ne citer qu'un exemple, il écrit :

Arriva un Huron nommé Tandhietsi des Trois-Rivières, qui apporta lettres de Montréal, de Richelieu et des Trois-Rivières, qui contenaient en substance qu'à Montréal tout se portait bien, et qu'il y avait quatre cabanes de Sauvages; qu'aux Trois-Rivières, il $y$ en avait douze, mais que le mélange des fidèles avec les infidèles et les apostats était toujours fâcheux...15

Québec était la résidence du Supérieur des Jésuites. Il était normal que celui-ci fût au courant de tout ce qui se passait dans la mission et qu'il insérât dans le Journal, aide-mémoire, ce qui lui serait utile pour la rédaction de la Relation.

Que de 1632 à 1645, il y ait eu un Journal des Jésuites de Québec, la chose ne fait pas de doute; et le père Jérôme Lalemant n'était pas sans le savoir. Quand il écrit Journal commencé, il n'entend pas introduire un usage absolument nouveau; il entend parler d'un registre qui fera désormais partie de la bibliothèque du Supérieur, et qui, tout en retenant les faits susceptibles d'être insérés dans la Relation, contiendra également les décisions prises par le Supérieur en vue du plus grand bien, les solutions apportées à des cas particuliers et difficiles, ainsi que les leçons dictées par l'expérience. Ce sera et un aide-mémoire en vue de la Relation et un aide-mémoire du Supérieur pour la bonne gouverne de la maison et de la mission, c'est-à-dire un coutumier.

Le père Jérôme Lalemant ne tarda pas à se rendre compte que, sous l'administration débonnaire du père Vimont, la résidence de Québec manquait de directives précises. Il y avait des coutumes, mais elles n'étaient consignées par écrit nulle part. Une expérience qu'il place dans le Journal à la fin d'octobre remonte aux premiers jours de son arrivée à Québec :

Arrivant ici on donnait la communion $1^{\circ}$ à M. le Gouverneur, et puis on s'en allait commencer par où il fallait; ne m'étant pas fait instruire là-dessus, la première fois je ne commençai pas par $M$. le Gouverneur.

Ayant été averti de la coutume, je commençai par lui la seconde fois; mais lui-même m'ayant dit qu'il en était choqué, je fis depuis comme la première fois, et ainsi il communie le dernier de la première tablée, à son rang selon la place où il se met 16 .

Il est évident que ce fait divers ne figurera pas dans la Relation; mais il a son utilité, il indique ce que sera désormais, sur ce point, la coutume.

15 Journal, 26.

16 Ibid., 12. 
De même la première entrée, celle du 17 octobre 1645, n'offrirait aucun intérêt aux lecteurs des Relations. Mais elle est d'un bon administrateur; elle fixe sur le papier un fait, dont l'oubli ou l'ignorance pourrait avoir des conséquences fâcheuses. Il s'agit du serviteur Chrétiennaut, envoyé comme cuisinier aux Trois-Rivières : " Il n'y eut point de marché par écrit avec lui ${ }^{17}$.,

Le père Lalemant note dans le Journal les objets qu'il a prêtés, par exemple : "Chapelle prêtée à $\mathrm{Mr}$ Nicolet, ptre, rendue et reprêtés ${ }^{18}$. C'est une coutume qu'il veut nnn nas introduire, mais consacrer, quand il écrit : "Il y eut grand'messe le jour de la Toussaint et le jour des Morts ${ }^{19}$.

Et pourquoi s'attarde-t-il à décrire minutieusement les grandes cérémonies de Noël et de la Semaine sainte, les processions de la FêteDieu et de l'Assomption? sinon pour établir une coutume conforme à la liturgie et couper par là aux fantaisies des individus. Il a soin de noter les erreurs commises par lui ou par d'autres, afin qu'on les évite à l'avenir; car en pareille circonstance, l'année prochaine, on recourra au Journal. Il écrit, le dimanche des Rameaux, 25 mars 1646 : "Pendant la Passion, je tenais mon rameau à la main gauche, ce qui ne faut pas, quand on la chante ${ }^{20}$. "Et le jeudi saint : "Les paradis furent faits; chez nous, au coin de l'autel, du côté de l'Epître; cela était bien. Il y eut quelque manque aux Hospitalières, en ce qu'il était en noir ${ }^{21}$. Voici enfin quelques leçons dictées par l'expérience : " On commença ici ce jour-là [le jeudi saint] la messe à 7 heures; il ne la faut commencer qu'à dix et demi ${ }^{22}$." Le samedi saint : "On commença une heure trop tôt; c'est assez de commencer à 9 heures, on commença à 8. On tira au Gloria quelques coups de canon; les Hospitalières sonnèrent sur les 9 heures, et hoc male"s.,

Second personnage de la colonnie, le Supérieur des Jésuites de Québec avait des obligations sociales à remplir, à l'occasion du Jour de l'An en particulier. Y avait-il sur le sujet quelque flottement? En tout cas, on aura désormais des indications précises. Le père Lalemant note les visites qu'il a faites, la qualité et la quantité des cadeaux qu'il a donnés, avec les noms des principaux bienfaiteurs amis ou domestiques, c'est-à-dire de ceux qu'il ne faut pas oublier : M. Giffard, le sympathique et zélé seigneur de Beauport, M. des Châtelets, secrétaire de la Compagnie des Habitants, l'ingénieur Bourdon, qui reçoit " une Lunette de Galilée, où il y avait une boussole ", celle qui lave le linge de l'église, $M$. et madame Abraham Martin, enfin le domestique

17

18

19 Ibid., 12. C'est le père Le Jeune qui avait établi l'usage des messes chantées, les dimanches et jours de fête. Voir plus bas Témoignage des Relations, année 1635-1636.

20

21

22

23

Journal, 39.

1 Ibid.

Ibid.

Ibid., 40. 
Robert Hache, à qui on avait destiné deux mouchoirs et qui en demanda - il fut exaucé - deux autres ${ }^{24}$ !

Le père Lalemant avait de la discipline religieuse une conception plus austère que le père Vimont. Celui-ci s'était permis des visites de pure civilité et des sorties dans le monde, qui paraissaient au père Lalemant incompatibles avec l'esprit religieux. Le redressement se fera lentement, avec prudence, mais il finira par s'imposer. En voici un exemple.

Le 4 novembre 1645 , donc un mois après son arrivée à Québec, le père Lalemant assiste avec le père Vimont au contrat de mariage de la fille de M. Giffard. * Nous y assistâmes, mais nous n'y signâmes point. M. le Gouverneur et plusieurs autres signèrent ${ }^{25}$. " $\mathrm{Ce}$ " nous n'y signâmes point » n'est pas l'effet du hasard; il indique chez le nouveau Supérieur une intention bien arrêtée. Si, après cela, il note la présence du père Vimont à des noces les 7,21 et 27 novembre, ce n'est pas qu'il approuve; il respecte tout simplement des engagements pris par son prédécesseur pendant qu'il était encore Supérieur. Mais voici que la chose se répète le 2 octobre 1646; et cette fois, le père Vimont est accompagné du père Le Jeune ${ }^{26}$. Le père Lalemant n'a pas jugé bon de refuser brusquement la permission à ses deux prédécesseurs. Mais il est temps de mettre fin à cet abus ! Et le 12 novembre, le père Vimont bénit le mariage de la veuve de Jean Nicolet avec Champagne. Ensuite de quoi, « il se délivre de l'importunité d'aller aux noces, et fait en sorte qu'on envoie de la noce quelque chose à la maison "27 ". Ce qu'il y a d'important ici, ce n'est pas que la veuve Nicolet ait pris mari; ce n'est pas que les Pères aient goûté à la noce tout en restant paisiblement chez eux, encore que le trait ne manque pas de saveur; ce qu'il y a de vraiment important, c'est que le père Vimont se soit a délivré * - on devine à l'instigation de qui - « de l'importunité d'aller aux noces ». Une telle rupture avec un passé tout récent méritait l'insertion dans le Journal. Elle marque une libération, elle exprime ce que sera désormais la coutume : on n'ira plus aux noces! C'est encore parce qu'il veut fixer la discipline domestique sur un point particulier, que le père Lalemant inscrit dans le Journal la fréquence et la matière des exhortations spirituelles données à la communauté ${ }^{28}$.

Il est bien permis de penser qu'en inscrivant dans son Journal les éléments d'un coutumier, le père Lalemant regarde plus loin et plus haut que Québec, qu'il vise à l'uniformité de la discipline dans toute la mission; cette uniformité devant venir de Québec, résidence du Supérieur. Aussi longtemps qu'il n'y avait eu qu'une résidence importante, celle de Québec, la nécessité d'un coutumier se faisait moins sentir. Mais les choses ont progressé rapidement en ces treize dernières années; 
et si la résidence de Québec garde un incontestable droit de priorité et de dignité, elle n'est plus la seule. Trois-Rivières grandit, Montréal paraît promis à un grand avenir, pendant les mois d'été, il y a des missionnaires résidents à Tadoussac et dans la lointaine Huronie il y a un personnel français important de donnés et de domestiques. Or, que deviendra l'unité de l'esprit s'il n'y a pas uniformité de discipline? Et puis, que dira ou que ne dira pas le monde, si les coutumes des Jésuites varient d'un endroit à l'autre, s'ils s'abstiennent d'aller aux noces à Québec pendant qu'ils y vont à Montréal ? L'heure est venue d'etabìır dans la ílission une même manière d'agir, d'y planies sulidement les bonnes coutumes, celles qui viennent de France et qui sont en même temps conforme à l'esprit de la Compagnie de Jésus. C'est à quoi s'applique le père Jérôme Lalemant.

L'élément coutumier existait-il dans le Journal du père Le Jeune? Nous ne le savons pas, puisqu'il avait déjà disparu quand le père Jérôme Lalemant entre en charge en 1645; d'autre part, notre seule source de reconstitution, la Relation, ne livre pas au grand public les petits problèmes d'ordre domestique. Mais quand le père Jérôme Lalemant écrit Journal commencé, il entend parler d'un cahier qui fera désormais partie de la bibliothèque du Supérieur. Il y a là des décisions, des solutions de cas particuliers, des leçons de l'expérience qui sont de nature à faciliter l'administration et à assurer une certaine uniformité de discipline dans toutes les missions de la Compagnie.

Son mérite, et la preuve que son Journal répondait à un besoin, c'est qu'il a été continué pendant plus d'un siècle par ses successeurs ou en leur nom ${ }^{29}$. Dans la seule partie qui nous est connue, on trouve en plus de son écriture, celle des pères Paul Ragueneau. François Le Mercier, Jean de Quen, Gabriel Druillettes, Pierre-Marie-Joseph Chaumonot et Simon Le Moyne, tous missionnaires de grand renom.

A tous, mais surtout au père Jérôme Lalemant, il faut, avant de finir, adresser un chaleureux merci. Car, nous avons assez parlé du Journal du père Lalemant, puisque ce n'est pas de lui qu'il s'agit directement ici. Encore fallait-il donner son véritable sens à l'expression Journal commencé et ne pas laisser libre cours à l'opinion, suivant laquelle il n'y aurait pas eu de Journal des Jésuites de Québec avant 1645. Redisons-le : celui que nous reconstituons ici a incontestablement existé; et il a la valeur que l'on doit reconnaître à ses rédacteurs, dont le principal n'est autre que le père Paul Le Jeune. Supérieur de Québec de 1632 à 1639 et auteur des onze premières Relations.

Léon Pouliot, s.j.

29 Le Journal, p. 199, note que du 10 février 1654 au 10 octobre 1656 , les entrées ont été faites dans un autre cahier, qui ne semble pas avoir été retrouvé. Les éditeurs du Journal, p. IX, nous apprennent que le père Vincent Bigot n'a pas tenu de Journal pendant son supériorat (1704-1710). 